TP Periodica Polytechnica

Chemical Engineering

58(Sup), pp. 21-26, 2014

DOI: $\underline{10.3311 / P P C h .7300}$

http://www.pp.bme.hu/ch/article/view/7300

Creative Commons Attribution (i)

RESEARCH ARTICLE

\section{The influence of surfactants to the stability of coal water suspension}

Saule Aidarova / Neila Bekturganova / Moldir Kerimkulova

Kuanyshbek Musabekov / Altynay Sharipova

RECEIVED 9 October 2012; ACcepted 28 MARCh 2013

\section{Abstract}

In this work a coal-water suspension with containing of $30 \%$ (mass) coal was investigated. Obtained coal-water suspension was stabilized by sodium dodecyl sulphate (SDS) and oxyethylated alkyl phenol (OP-10). A wettability isotherm of coal surface by surfactants, optimal regime of coal dispersion, adsorption isotherm, viscosity, fluidity and heat of combustion of $30 \%$ (mass) coal-water suspension were determined. Obtained results allow to recommend 30\% coal-water suspension stabilized by surfactants as a fuel.

\section{Keywords}

wetting $\cdot$ adsorption $\cdot$ rheology $\cdot$ stability $\cdot$ combustion heat

\section{Saule Aidarova}

International Postgraduate Institute "Excellence PolyTech" of Kazakh National

Technical University named after K. Satpaev, Satpaev str. 22, Almaty, Kazakhstan Altynay Sharipova

International Postgraduate Institute "Excellence PolyTech" of Kazakh National Technical University named after K. Satpaev, Satpaev str. 22, Almaty, Kazakhstan e-mail: altynay.sharipova@mpikg.mpg.de

\section{Neila Bekturganova}

Department of Applied Chemistry,

Kazakh National Technical University named after K. Satpaev,

Satpaev str. 22, Almaty, Kazakhstan

Moldir Kerimkulova

Kuanyshbek Musabekov

al-Farabi Kazakh National University, al-Farabi av. 71, Almaty, Kazakhstan

\section{Introduction}

The stable tendency of a price growth of oil products leads to an intensive research of a new technology of a coal refining because the explored coal reserves are 20 times more than oil reserves. Coal-water suspensions are paid attention as a real alternative to liquid fuels from oil in the countries such as Japan, People's Republic of China, Italy, USA, Sweden and Russian Federation. This is connected with a high scientific and technical potential gathered by these countries in the area of production, transportation, burning of coal-water fuels in boiler, cogeneration plants, combined-cycle plants and gas turbines [1-8].

However, despite of these advantages of a coal-water fuel, there are several disadvantages, among which the most important is the instability of a liquid fuel. It is known that the violation of stability, sedimentation of particles and the lamination of the dispersed system are due to the differences in specific gravity of the dispersed phase and a liquid dispersion medium [6]. Therefore for the preparation of coal-water suspensions from a theoretical point of view is advisable to use the fractions with the same (proportional) size of coal particles [9-12].

Another important way to improve the stability of coal-water suspension (CWS) is the addition of stabilizers (polymers and surfactants) that reduce the coagulation (coalescence) of coal particles and their sedimentation [13-17].

In addition, in accordance to fundamental principles of physicochemical mechanics and the modern theory of the stability of dispersed systems (theory of Derjaguin-Landau-VerweyOverbeek) the stabilization of the dispersion is determined by the potential curve of the dispersed phase (DP) interaction. In particular, the coagulation interaction in dispersions at the concentrations of dispersed phase is higher than the critical micelle concentration depends on the nature of the particles of dispersed phase, temperature, ionic strength and the presence of low and high molecular surfactants.

It is also known that the stability of dispersed systems is determined by the surface properties of the dispersed phase. It is known that the coal surface is hydrophobic so surfactants with different nature have higher affinity to the coal surface. 
The purpose of this work is to study of the stability of $30 \%$-(mass.) water-coal suspension in the presence of anionic surfactant sodium dodecyl sulphate (SDS), nonionic surfactant oxyethylated alkylphenol OP-10 and the determination of thermophysical properties (heat of combustion) of the obtained composite system.

\section{Experimental}

As an object coal with a moisture $\mathrm{W}^{\mathrm{a}}-7.7 \%$; ash content $\mathrm{A}^{\mathrm{a}}-13.67 \%$; volatile matter content $\mathrm{V}^{\text {daf }}-43.3 \%$; carbon content in a dry form Cdaf- $73.89 \%$ was chosen. All experiments were performed at $\mathrm{t}(22 \pm 0.1)^{\circ} \mathrm{C}$. The error of wetting was 10 , the error of the adsorption at the solid/fluid - 0.1-0.2\%, whereas the error for the sedimentation measurements was equaled to \pm 0.30 units in eyepiece.

Particle with the sizes in the range 0.2-0.5 microns were obtained by dispersing in the colloid mill. By sifting through a sieve with a pore size of $0.25 \mathrm{~mm}$ a fraction up to size $<0.25$ $\mathrm{mm}$ was separated which was used in further studies. The particle size of coal and sedimentation analysis was determined by Figurovskyi method which was described elsewhere [17]. First experiments according to method [17] were carried to determine the size of the dispersed phase, and then from the obtained data the path $(\mathrm{N})$ of deposited particles after mixing was defined. According to it the sedimentation rate and particle radius were determined:

$$
\mathrm{U}_{\mathrm{i}}=\mathrm{H} / \mathrm{t}_{\mathrm{i}}
$$

where $t_{i}$ - the time which neads to finish to sedimentate a given fraction, min; $\mathrm{H}$ - distance of the dispersed phase from the liquid surface to the bottom of the cup, $\mathrm{cm}$.

After the determination of the sedimentation rate the radius $\left(r_{i}\right)$ of particles in this fraction was found:

$$
r_{i}=\sqrt{c U_{i}}
$$

where $\mathrm{c}$ - constant value equal to

$$
c=\frac{9}{2} \frac{\eta}{\left(d_{s}-d_{f}\right) g}
$$

$\mathrm{d}_{\mathrm{s}}-$ density of the solid dispersed phase;

$\mathrm{d}_{\mathrm{f}}$ - the density of the fluid;

$\eta$ - coefficient of viscosity of the medium;

$\mathrm{g}$ - acceleration of gravity.

The obtained aggregative unstable hydrosuspension was stabilized with surfactants. Anionic surfactant sodium dodecyl sulphate (SDS) with molecular weight $\mathrm{M}=288$, nonionic surfactant oxyethylated phenol OP-10 with molecular weight $M=646$ were used to stabilize the hydrosuspension. Aqueous solutions of polymers with a concentration in the range of 0.1-1.0\% of monomer units of polymer were used.
Contact angle was determined by goniometer and horizontal microscope with a movable tumbler [18]. Contact angle was found on basic sizes (height $h$ and radius of a basis $r$ ) of liquid drops on the surface. $\operatorname{Cos} \theta$ values were calculated by formulas:

$$
\begin{aligned}
& \text { In case of } \theta<90 \quad \cos \theta=\frac{r^{2}-h^{2}}{r^{2}+h^{2}} \quad \theta=1-\frac{h}{2} \\
& \text { In case of } \theta>90 \quad \cos \theta=1-\frac{h}{2}
\end{aligned}
$$

where $\mathrm{h}$ and $\mathrm{r}$ - parameters determined by ocular micrometer.

$$
\mathrm{r}=\mathrm{d} / 2
$$

$\mathrm{d}$ - the diameter of droplet basis deposited on the Gonio wafer; $\mathrm{h}$ - the height of drop of fluid determined by the formula

$\mathrm{h}=\left(\mathrm{n}_{2}-\mathrm{n}_{1}\right)$,

where $\mathrm{n}_{2}$ and $\mathrm{n}_{1}-$ ocular division.

\section{Experimental results}

For the preparation of a stable hydrosuspension highly dispersed coal particles were obtained. Particle size distribution of dispersed coal particles is shown in figure 1 .

Coal dispergation was carried up to 120 minutes, in this case the polydispersed coal particles were formed. Despite the fact that maximum of the particle size distribution $(26.9 \%)$ is in the range of radii $8.0-8.4 \mu \mathrm{m}$, the other particles radii are in the range of $0.3-50 \mu \mathrm{m}$.

The dispergation time increase up to 180 minutes did not show significant influence to the particles dispersity. That is why for further investigations dispergation time was kept 120 minutes.

According to the physico-chemical mechanics laws of the highly dispersed particles obtaining the dispergation would be better with the presence of surfactants. That is why as surfactants sodium dodecyl sulphate (SDS) and nonionic surfactant oxyethylated phenol (OP-10) were used.

For the selection of surfactants optimal concentration the wetting isotherms of coal surface by the aqueous solutions of surfactants were obtained. With increasing of surfactant concentration the significant decrease of contact angles of coal surface by SDS ( from $65^{\circ}$ up to $15-28^{\circ}$ ) was observed (Figure 2).

Note that the adsorbed surfactant molecules do not only reduce the coal durability but also increase the aggregate stability of its suspension in water. According to the well-known thermodynamic relation for the highly dispersed systems the reduce of the surface tension leads to a decrease of the free energy and stability increase:

$$
\mathrm{F}=\sigma \mathrm{S}
$$

where F-Helmholtz free energy; $\sigma$ - surface tension of the solid; $\mathrm{S}$ - surface area of the solid. 
Thus, on the basis of the experimental results the optimal regime of coal dispergation - dispergation of preliminary crushed coal in wet state in the presence of $0.8 \%$ OP- 10 or $0.4 \%$ SDS for 120 minutes was established.

For the preparation of suspensions the effect of dispersed phase concentration on the lifetime of a water-coal suspension was investigated. It was found that the increase of the coal concentration from $10 \%$ to $50 \%$ leads to an increase of the stability (Figure 3).

The stability of the coal-water suspension was controlled by reducing the sedimentation rate of dispersed particles. The growth of the kinetic (sedimentation) stability of concentrated $(40 \%-50 \%)$ coal suspensions is probably due to the formation of coagulation structures in them. From this perspective the concentrated coal suspensions are useless to study the effects of various factors on the stability and rheological properties. Therefore for further investigation the $30 \%$ of coal-water suspension was chosen which are sensitive to the influence of external factors (surfactant and polymer additives, the method of suspension, etc.).

To establish the effect of hydrophilization and electrostatic coal surface charge on the stability of the coal-water suspension the kinetics of sedimentation of 30\% coal-water suspension in the presence of OP-10 and SDS was investigated.

Small additions of these surfactants did not have a significant effect on the stability of the coal-water suspension (Figure 4), only when the concentration is close to the critical micelle concentration $(0.2-0.4 \%)$ a stabilizing effect of these surfactants on the coal-water suspension is started.

The experimental results are shown for the $\mathrm{CMC}(\mathrm{OP}-10)$ $\mathrm{C}=0.4 \%$ and $\mathrm{CMC}(\mathrm{SDS})=0.2 \%$. The study of the effect of these surfactants to stabilize the WCS at lower concentrations of OP-10: $0.1,0.15,0.2,0.25,0.3 \%$ and in the case of SDS 0.02 , $0.05 \%$ showed complete destabilization of water-coal suspension in 15-50 minutes. This is most likely explained by insufficient amount of the stabilizer in the system. Free location of surfactant macromolecules on the surface of coal particles promotes to the binding together by hydrophobic interactions.

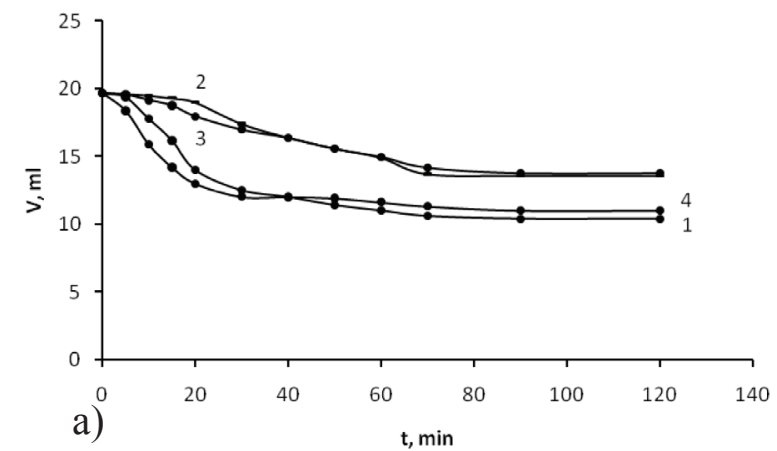

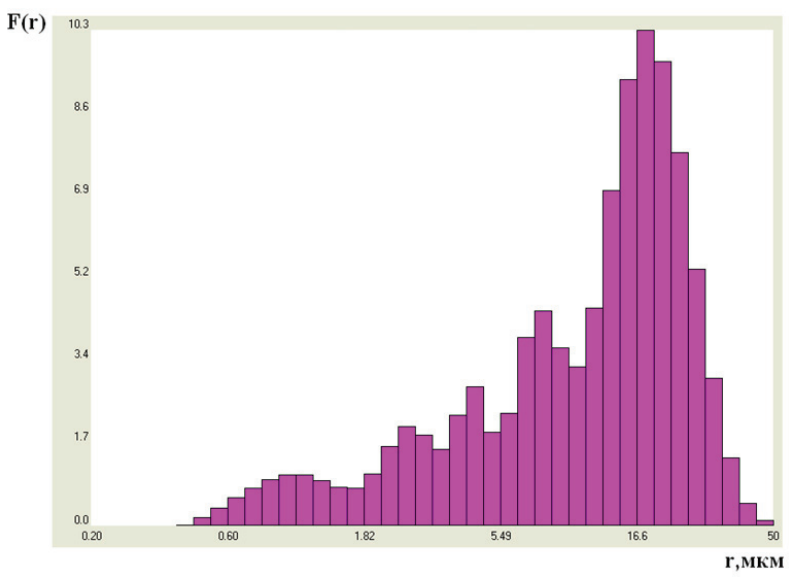

Fig. 1. Size distribution of dispersed coal particles.

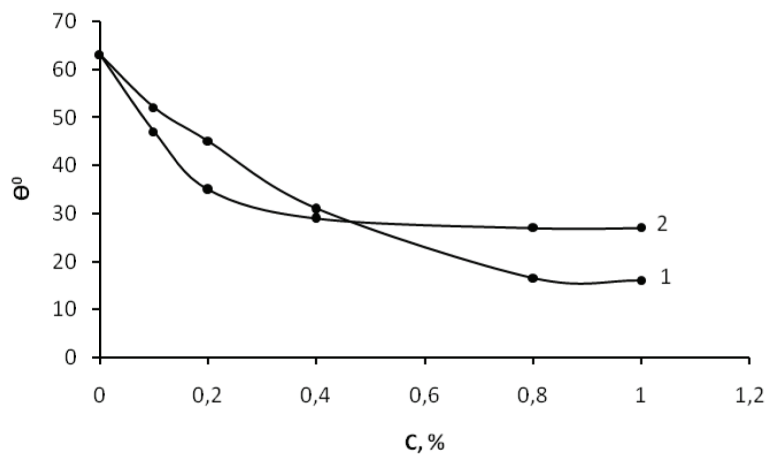

Fig. 2. Wetting isotherm of coal surface by OP-10 (1), SDS (2).

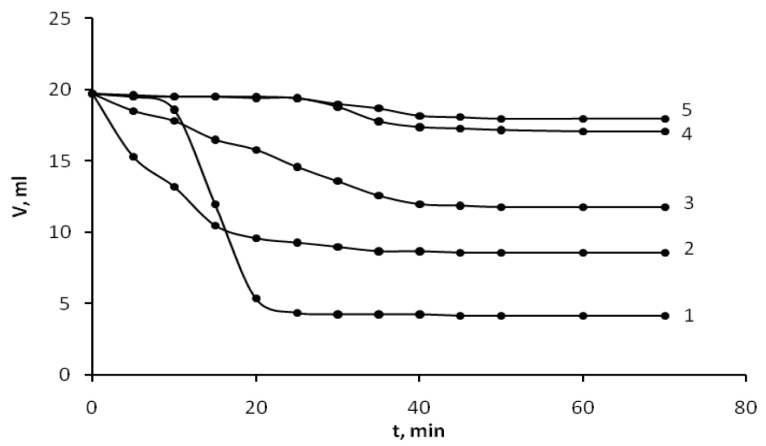

Fig. 3. The sedimentation of particles from its $10 \%$ (1), $20 \%(2), 30 \%$ (3), $40 \%$ (4) and $50 \%(5)$ of the suspension.

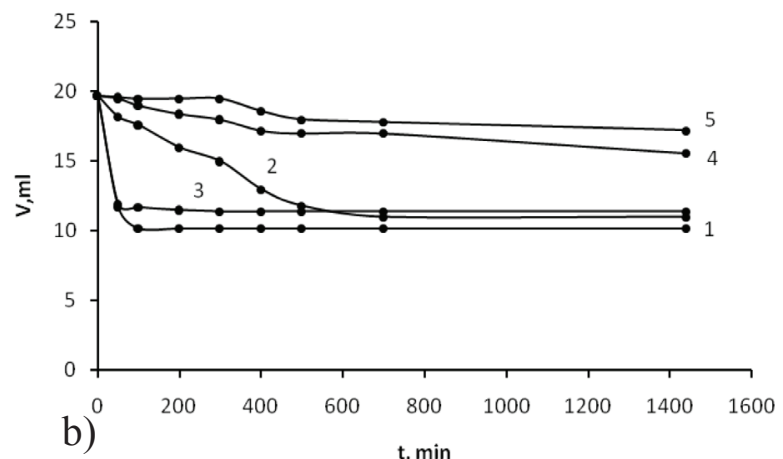

Fig. 4. The kinetics of the sedimentation of $30 \%$ hydrosuspension in the presence of OP-10 (a) and SDS (b). a) $\mathrm{COP}_{-10}: 0.1 \%(1), 0.4 \%(2), 0.8 \%(3), 1.0(4)$., b) $\mathrm{C}_{\mathrm{SDS}}: 0.1 \%(1), 0.2 \%(2), 0.4 \%(3), 0.8 \%(4), 1.0 \%(5)$. 
This leads to a rapid aggregation of particles, it means to appearance of large aggregates which consist of ten to hundreds of particles.

The stabilization of dispersed systems in the presence of surfactants in the region of CMC can be interpreted by the fact that in case of the adsorption of surfactants on the coal surface the interaction occurs on the hydrophobic parts of coal surface and hydrocarbon radicals of surfactant molecules of. The mutual repulsion of similarly charged particles leads to the stabilization of coal suspension. However to achieve a such state the coal suspension goes through a series of intermediate stages which is due to the different position of the surfactant molecules in the adsorbed layer.

To determine the mechanism of the stabilizing effect of surfactants their adsorption on the coal surface was studied (Figure 5).

The adsorption isotherm OP-10 corresponds to the S-shaped multilayer adsorption isotherm. As the calculations showed in the investigated concentration range $(<1.0 \%)$ on the coal surface the adsorption layers of surfactants which are closed to the saturation are formed. As the capacity of a saturated monomolecular adsorption layer OP-10 is $\mathrm{A}_{\infty} \approx 7.0 \cdot 10^{-3} \mathrm{mg} / \mathrm{g}$ (Figure 5) it is possible to calculate the degree of the coating of a coal surface by surfactant molecules: for the coal concentrations $0.4 \%$, $0.8 \%$ and $1.0 \%$ the concentration of OP-10 solutions is $80 \%$, $90 \%$ and $140 \%$, respectively. The similar results were obtained in the investigation of SDS adsorption.

For more information on the surfactant adsorption on coal the infrared spectra of the coal particles with adsorbed molecules of the OP-10 and SDS was studied. The intensity of the absorption bands of the $\mathrm{O}-\mathrm{H}$ valent fluctuations bond for the adsorbed molecules is more $\left(3428 \mathrm{~cm}^{-1}\right)$ than for free but a structured absorption band is higher. The position of this band does not change in the spectra for the systems formed by these reagents. This indicates the absence of the interaction. In the spectra of the OP-10 absorption bands which indicates the hydroxyl group are observed at 1620 and $1616 \mathrm{~cm}^{-1}$. The visible absorption band is related to the covalent fluctuations of $\mathrm{C}=\mathrm{C}$ bond of aromatic ring.

The spectra of the SDS shows the absorption bands of deformation valent vibrations of the $\mathrm{C}-\mathrm{H}$ bond. Although the intensity is not high, the band can be seen well in all cases (Figures 6,7).

Thus, with increasing of the surfactant concentration in the hydrosuspension on the coal surface the multilayer adsorption layer is formed. The formation of a such saturated adsorption layer increases the strong stabilizing factor for dispersed systems (Rehbinder's structural-mechanical factor).

As the ultimate objective of the study was to prepare a sustainable coal-water fuel for the hydrotransportation and direct combustion in boilers, furnaces and other objects of power plants important thermal characteristics of coal-water suspensions such as combustion heat (Q) was studied for using of coal-water suspensions in practice (Table 1).

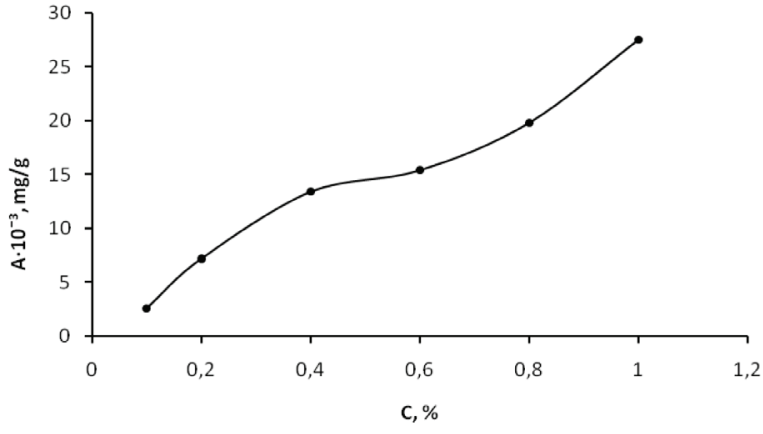

Fig. 5. The adsorption isotherm of OP-10 on the surface of coal particles.

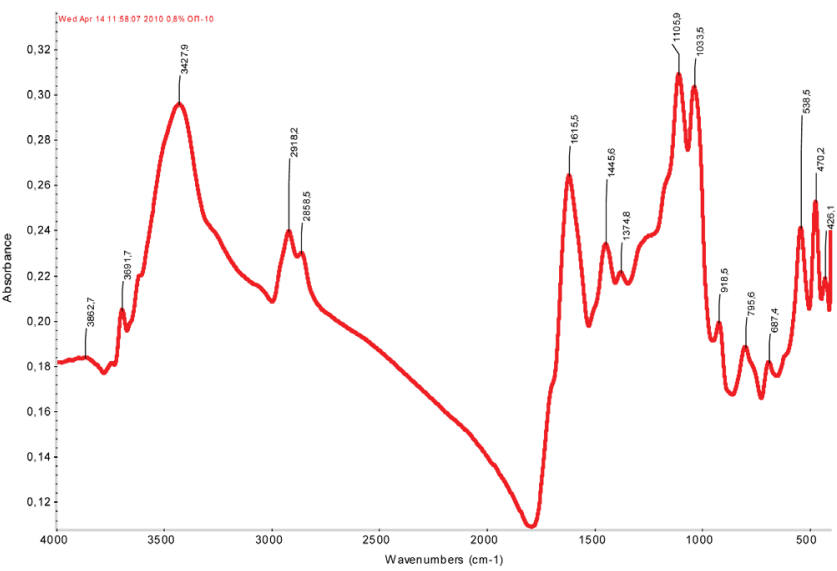

Fig. 6. IR-spectra of coal modified by $0.8 \% \mathrm{OP}-10$

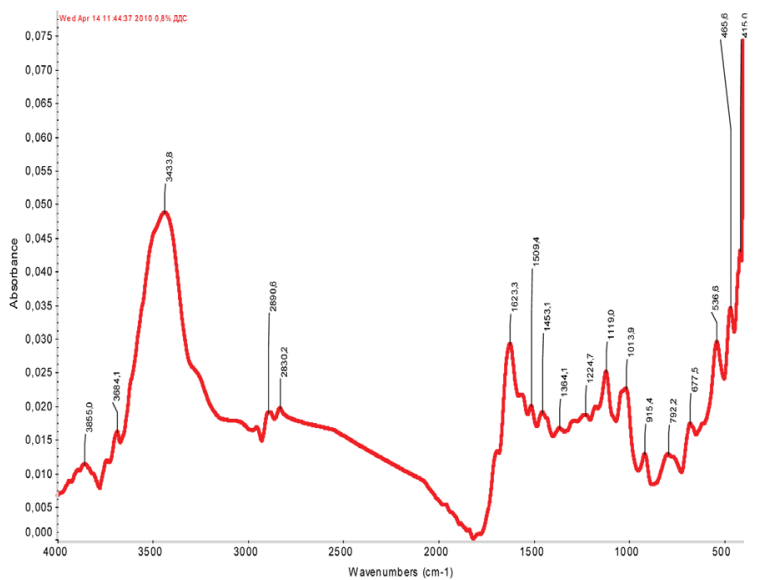

Fig. 7. IR-spectra of coal modified by $0.8 \%$ SDS

Table 1 shows that the fuel characteristics of the coal-water suspension do not worse than similar characteristics of pure coal. In some cases, for example for $30 \%$ coal-water suspensions stabilized by composition of $0.8 \%$ OP-10 the heat of combustion values superior to those for dry coal. This phenomenon has been observed for the first time. It can be interpreted by the dissociation of water molecules at $900^{\circ} \mathrm{C}$ for $\mathrm{H}+$ and $\mathrm{OH}^{-}$ions which participate in the coal combustion and reduce the combustion temperature $\left(300-400^{\circ} \mathrm{C}\right)$ of fuel. 
Tab. 1. Results of the calorimetric determination of combustion heat of coal and its hydrosuspensions.

\begin{tabular}{|c|c|c|c|c|c|}
\hline Sample & $\begin{array}{c}\text { The mass of } \\
\text { combustible components } \\
\text { in the sample, } \mathrm{g}\end{array}$ & $\begin{array}{c}\text { Value of } \\
\Delta \mathrm{t},{ }^{\circ} \mathrm{C}\end{array}$ & $\begin{array}{c}\text { Average value } \\
\Delta \mathrm{t}_{\text {average }}\end{array}$ & $\begin{array}{l}\text { Average value } \\
\qquad \mathrm{Q}_{\mathrm{av}}, \mathrm{kJ}\end{array}$ & $\begin{array}{l}\text { Value } \\
Q_{a v}, k J .\end{array}$ \\
\hline Pure coal & 4.0 & $\begin{array}{l}9.21 \\
9.25 \\
9.20\end{array}$ & 9.22 & 102.15 & 25539.4 \\
\hline $30 \%$ coal-water suspension & 1.2 & $\begin{array}{l}2.7 \\
2.5 \\
2.6\end{array}$ & 2.6 & 28.81 & 24006.7 \\
\hline $30 \%$ coal-water suspension stabilized by $1.0 \%$ SDS & 1.24 & $\begin{array}{l}2.67 \\
2.67 \\
2.70\end{array}$ & 2.68 & 29.7 & 23947.1 \\
\hline $30 \%$ coal-water suspension stabilized by $0.8 \%$ OP- 10 & 1.23 & $\begin{array}{l}2.90 \\
2.87 \\
2.87\end{array}$ & 2.88 & 31.9 & 25901.3 \\
\hline
\end{tabular}

\section{Conclusions}

In this work the coal-water suspension with containing of $30 \%$ (mass) of coal was investigated. The obtained coal-water suspension was stabilized by sodium dodecyl sulphate (SDS) and oxyethylated alkyl phenol (OP-10). Based on the experimental results it was established that the best wettability have $0.8 \%$ OP-10 and $0.4 \%$.
Optimal regime of coal dispergation as dry crushed coal dispergation in wet state in the presence of surfactants was determined. It was found that the stabilizing effect of OP-10 and SDS on the coal-water suspensions appears at surfactant concentrations close to the critical micelle concentration (0.2-0.4\%). The adsorption isotherms of surfactants corresponds to S-shaped multilayer adsorption isotherm.

\section{Acknowledgements}

The work was done in the frame of Ministry of Education and Science of Republic of Kazakhstan project entitled "Development of new type of coal-water fuel on the basis of Kazakhstani coal".

\section{References}

1 Makarov A. S., Klishchenko R. E., Zavgorodnii V. A., Makarova E. V., The Impact of the Water Salt Content on the Properties of CoalAqueous Suspensions. Journal of Water Chemistry and Technology, 33(6), 357-362 (2011).

DOI: $10.3103 / \mathrm{S} 1063455 \mathrm{X} 11060026$

2 Kijo-Kleczkowska A., Combustion of coal-water suspensions. Fuel, 90(2), 865-877 (2011). DOI: $10.1016 /$ j.fuel.2010.10.034

3 Zhou M., Pan B., Yang D., Lou H., Qiu X., Rheological Behavior Investigation of Concentrated Coal-Water Suspension. Journal of Dispersion Science Technology, 31(6), 838-843 (2010). DOI: $10.1080 / 01932690903333788$

4 Laskowski J. S., Coal Flotation and Fine Coal Utilization. Elsevier, Amsterdam (2002).

5 Redlich P. J., Jackson W. R., Larkins F. P., Chaffee A. L., Krichko A. A., Grigor'eva E. A., Shatov S. N., Comparison of the structure and reactivity of a Kansk-Achinsk basin (USSR) coal with those of a Latrobe Valley (Australia) coal. Energy and Fuels, 4(1), 28-33 (1990). DOI: 10.1021/ef00019a006

6 Tamon H., Izhizaka H., Yamamoto T., Suzuki T., Preparation of mesoporous carbon by freeze drying. Carbon, 37(12), 2049-2055 (1999).

7 Khodakov G. S., Gorlov E. G., Golovin G. S., Production and Pipeline Transportation of Coal-Water Slurry Fuel. Chemistry of solid fuels, 40(4), 19-35 (2006).

8 Baranova M. P., Kulagin V. A., Taraban'ko V. E., Nature of Stabilization of Water-Coal Fuel Suspensions. Russian Journal of Applied Chemistry, 84(6), 939-944 (2011). DOI: $10.1134 / \mathrm{S} 1070427211060073$

9 Boylu F., Atesok G., Dincer H., Effect of coal particle size distribution, volume fraction and rank on the rheology of coal-water slurries. Fuel Processing Technology, 85(4), 241-250 (2004). DOI: 10.1016/S0378-3820(03)00198-X

10 Cheng J., Zhou J., Li Y., Liu J., Cen K., Effects of pore fractal structures of ultra fine coal water slurries on rheological behavior and combustion dynamics. Fuel, 87(12), 2620-2627 (2008). DOI: $\underline{10.1016 / \mathrm{j} . f u e l .2008 .01 .028}$

11 Nunez G.A., Briceno M.I., Joseph D.D., Asa T., Gomez C., Nano-Dispersions of Coal in Water for Use as a Fuel and Methods of Making Same. US 2011/0203163 A1, USA (2011).

12 Chanson H., Gaolin Q., Drag reduction in hydraulics flows. in '1994 International conference on Hydraulics in Civil Engineering. University of Queensland-Brisbane, Australia' 94, 123-128 (1994).

13 Li P., Yang D., Lou H., Qiu X., Study on the stability of coal water slurry using dispersion-stability analyzer. Journal of Fuel Chemistry and Technology, 36(5), 524-529 (2008). DOI: $10.1016 / \mathrm{S} 1872-5813(08) 60033-\mathrm{X}$

14 Lee S., Speight J. G., Loyalka S. K., Coal Slurry Fuel. in 'Handbook of Alternative Fuel Technologies' Taylor \& Francis Group, New York, 125-152 (2007). 
15 Tudor P. R., Atkinson D., Crawford R. J., Mainwaring D. E., The effect of adsorbed and non-adsorbed additives on the stability of coalwater suspensions. Fuel, 75(4), 443-452 (1996).

DOI: $\underline{10.1016 / 0140-6701(96) 88464-5}$

16 Tadros T. F., Taylor P., Bognolo G., Influence of addition of a polyelectrolyte, nonionic polymers, and their mixtures on the rheology of coal/water suspensions. Langmuir, 11(12), 4678-4684 (1995).

DOI: $\underline{10.1021 / 1 \mathrm{a} 00012 \mathrm{a} 017}$
17 Kouzov P. A., Basics of analysis of disperse composition of industrial dusts and crushed materials. L.:Chemistry, 264 (russ) (1987).

18 Voyutsky S. S., The course of colloid chemistry. M.:Chemistry, 512 (russ) (1975). 